\title{
Shaping opinions in a social network
}

\author{
T. Carletti* and S. Righi \\ Département de Mathématique, Facultés Universitaires Notre-Dame de la Paix \\ Namur, Belgium, 5000 \\ *E-mail: timoteo.carletti@fundp.ac.be \\ www.fundp.ac.be
}

\begin{abstract}
We hereby propose a model of opinion dynamics where individuals update their beliefs because of interactions in acquaintances' group.

The model exhibit a non trivial behavior that we discuss as a function of the main involved parameters. Results are reported on the average number of opinion clusters and the time needed to form such clusters.
\end{abstract}

Keywords: sociophysics; opinion dynamics; agent based model; group interactions

\section{Introduction}

Complex Systems Science (CSS) studies the behavior of a wide range of phenomena, from physics to social sciences passing through biology just to mention few of them. The classical approach followed in the CSS consists first in a decomposition of the system into "elementary blocks" that will be successively individually analyzed in details, then the properties determined at micro-level are transported to the macro-level. This approach results very fruitful and shaped the CSS as an highly multidisciplinary field.

Recently models of opinion dynamics gathered a considerable amount of interest testified by the production of specialized reviews such as 1-3, reinforcing in this way the emergence of the sociophysics. ${ }^{4} \mathrm{~A}$ basic distinction can be done in model of continuous opinion with threshold, ${ }^{5,6}$ where opinions can assumed to be well described as continuous quantities; thus agents update their values because of binary interactions, if their opinions are close enough, i.e. below a given threshold. The second class consists of models where opinions can be described by discrete variable, yes/no for instance, and they are updated according to local rules, i.e. small group interactions as for instance: majority rule, ${ }^{7}$ majority and inflexible rules, ${ }^{8}$ 
majority and contrarian rules. ${ }^{9}$

In this paper we introduce a new model of opinion dynamics which naturally sets at the intersection of the former scheme; in fact individuals have continuous opinions that are updated if they are below some given threshold, once agents belong to a group, whose size evolves dynamically together with the opinion. Moreover each agent possesses an affinity with respect to any other agent, the higher is the affinity score the more trustable is the relationship. Such affinity evolves in time because of the past interactions, hence the acquaintances' group is determined by the underlying evolving social network.

We hereby provide an application of the model to the study of the consensus-polarization transition that can occur in real population when people do agree on the same idea - consensus state - or they divide into several opinion groups - polarization state.

The paper is organized as follows. In the next section we introduce the model and the basic involved parameters, then we present the results concerning the consensus-polarization issue and the time needed to reach such asymptotic state. We will end with some conclusions and perspectives.

\section{The model}

The model hereby studied is a generalization of the one proposed in ${ }^{6,10}$ because now interactions occur in many-agents groups, whose size is not fixed a priori but evolves in time.

We are thus considering a fixed population made of $N$ agents, i.e. closed group setting, where each agent is characterized by its opinion on a given subject, here represented by a real number $O_{i}^{t} \in[0,1]$, and moreover each agent possesses an affinity with respect to any other, $\alpha_{i j}^{t} \in[0,1]$ : the higher is $\alpha_{i j}^{t}$ the more affine, say trustable, the relationships are and consequently agents behave.

At each time step a first agent, say $i$, is randomly drawn with an uniform probability, from the population; then, in order to determine its acquaintances' group, it computes its social distance with respect to the whole population:

$$
d_{i j}^{t}=\left|O_{i}^{t}-O_{j}^{t}\right|\left(1-\alpha_{i j}^{t}\right), \forall j \in\{1, \ldots, N\} \backslash\{i\} .
$$

The agents $j$ whose distance from $i$ is lesser than a given threshold, $\Delta g_{c}$, will determine the acquaintances' group of $i$ at time $t$ in formula:

$$
\mathcal{F}_{i}^{t}=\left\{j: d_{i j}^{t} \leq \Delta g_{c}\right\}
$$


Let us observe that the group changes in time, in size and in composition, because the opinions and/or affinities also evolve. The rationale in the use of the affinities in the definition of the social metric is to interpret ${ }^{10,11}$ the affinity as the adjacency matrix of the (weighted) social network underlying the population. We hereby assume a constant threshold $\Delta g_{c}$ for the whole population, but of course one could consider non-homogeneous cases as well.

Once the agent $i$ has been selected and the group $\mathcal{F}_{i}^{t}$ has been formed, the involved individuals do interact by possibly updating their opinions and/or affinities. Once in the group, all agents are supposed to listen to and speak to all other agents, therefore every one can perceive a personal averaged - by the mutual affinity - group opinion, $\left\langle O_{l}^{t}\right\rangle$, because each agent weights differently opinions of trustable agents from the others. In formula:

$$
<O_{l}^{t}>=\frac{\sum_{j=1}^{m_{i}} \alpha_{l j}^{t} O_{j}^{t}}{\sum_{j=1}^{m_{i}} \alpha_{l j}^{t}} \quad \forall l \in \mathcal{F}_{i}^{t},
$$

where we denoted by $m_{i}$ the size of the subgroup $\mathcal{F}_{i}^{t}$. The vector $\left(<O_{1}^{t}\right\rangle$ $, \ldots,<O_{m_{i}}^{t}>$ ), will hereby named apopsicentre, i.e. the barycentre of the opinions $(\alpha \dot{\alpha} \sigma \psi \eta \eta$ opinion).

Because the affinity is is general not symmetric, some agents could have been included in the group determined by $i$, "against" their advise, hence a second relevant variable is the averaged affinity that each agent perceives of the group itself ${ }^{a}$ :

$$
<\alpha_{l}^{t}>=\frac{1}{m_{i}} \sum_{j=1}^{m_{i}} \alpha_{l j}^{t} \quad \forall l \in \mathcal{F}_{i}^{t} .
$$

Once the former two quantities have been computed by each agent, we propose the following update scheme: to belong in the largest size group, each agent, would like to come closer to its perceived apopsicentre if it feels himself affine enough to the group:

$$
O_{l}^{t+1}=O_{l}^{t}+\frac{1}{2}\left(<O_{l}^{t}>-O_{l}^{t}\right) \Gamma_{1}\left(<\alpha_{l}^{t}>\right) \quad \forall l \in \mathcal{F}_{i}^{t},
$$

where $\Gamma_{1}(x)=\frac{1}{2}\left[\tanh \left(\beta_{1}\left(x-\alpha_{c}\right)\right)+1\right]$ is an activating function, defining the region of trust for effective social interactions, e.g. $\left\langle\alpha_{l}^{t}>\right.$ larger than $\alpha_{c}$.

${ }^{\mathrm{a}}$ Each agent in the group can determine the apopsicentre, but if it is in an hostile group, it will not move toward this value. 
Moreover sharing a close opinion, reinforce the mutual affinity, while too far opinions make the relationship to weak, hence each agent becomes more affine with all the agents in the subgroup that share opinions close enough to its perceived apopsicentre, otherwise their affinities will decrease:

$$
\alpha_{j k}^{t+1}=\alpha_{j k}^{t}+\alpha_{j k}^{t}\left(1-\alpha_{j k}^{t}\right) \Gamma_{2}\left(\Delta O_{j k}\right) \quad \forall j, k \in \mathcal{F}_{i}^{t},
$$

where $\Delta O_{j k}=<O_{j}^{t}>-O_{k}^{t}$, and

$$
\Gamma_{2}(x)=\tanh \left[\beta_{2}\left(\Delta O_{c}-|x|\right)\right],
$$

that can be considered again as an activating function for the affinity evolution. In the previous relations for $\Gamma_{1,2}$, we set the parameters $\beta_{1}$ and $\beta_{2}$, large enough to practically replace the hyperbolic tangent with a simpler step function. Under these assumptions $\Gamma_{1}$ takes values either 0 or 1 , while the value of $\Gamma_{2}$ are either -1 or 1 . The interaction mechanism is schematically represented in Fig. 1.

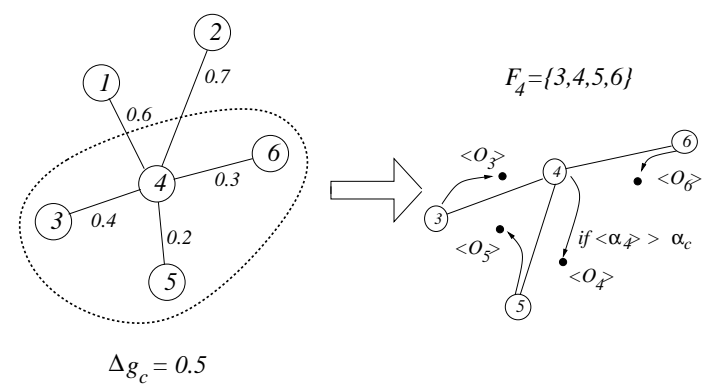

Fig. 1. Cartoon to represent the group formation and the interaction mechanism. On the left panel, the composition of the local acquaintance group. On the right panel, dynamics in the opinion space : each agent tends to move following the represented arrows.

\section{Results}

A typical run of this model is presented in Fig. 2. First of we can observe that the dynamics is faster than in the similar model presented in 6,10 , this is because binary interactions are replaced by multi-agents interactions that improve the information spread. Moreover there exists a transient interval of time, where nobody modifies its opinion, but only the mutual affinities (see insets of Fig. 2 and the relative caption). Only when the relationships become highly trustable, agents do modify also their opinions. 
This behavior is explained by the different time scales of the main processes: evolution of opinions and evolution of affinity, as it clearly emerges again from the insets of Fig. 2, where we show three time-snapshots of the affinity, i.e. the social network, once agents start to modify their opinions. Transferring this behavior to similar real social experiment, we could expect that, initially unknown people first change (in fact construct) their affinities relationships (increasing or decreasing mutual affinities) and only after that, they will eventually modify their opinions. Namely initially people "sample" the group and only after they modify their beliefs.

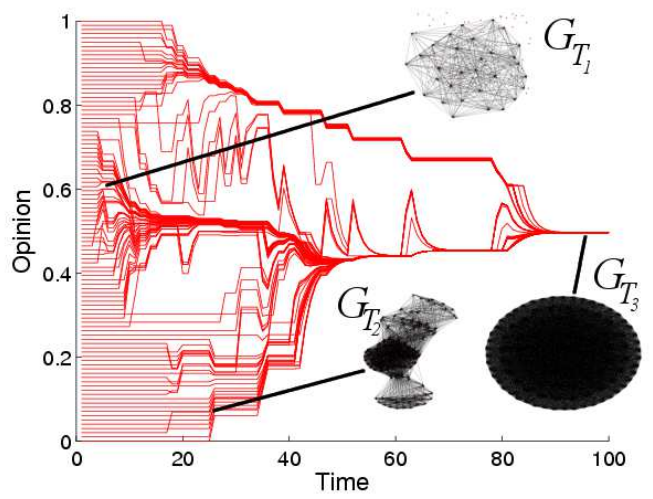

Fig. 2. Time evolution of the opinion (main panel) and the social network of affinity time-snapshots (small insets). Parameters are: $\Delta g_{c}=0.1, \alpha_{c}=0.5, \Delta O_{c}=0.5, N=100$ agents whose opinion are initially uniformly distributed in $[0,1]$, whereas initial affinities are uniformly distributed in $[0,1 / 2]$. Three time-snapshot of social networks are reported for increasing times, $T_{3}>T_{2}>T_{1}$. Dots represent agents that are possibly linked if they are affine enough. Each network has been characterized by some standard topological indicator; the diameter and the averaged shortest path (that are infinite for $G_{T_{1}}$ and respectively have values 4 and 1.81 for $G_{T_{2}}$ and 2 and 1.00 for $G_{T_{3}}$ ), the averaged degree (that takes values $0.07,0.39$ and 0.9 respectively for $G_{T_{1}}, G_{T_{2}}$ and $G_{T_{3}}$ ) and the averaged network clustering (that assumes the values $0.10,0.72$ and 0.99 respectively for $G_{T_{1}}, G_{T_{2}}$ and $G_{T_{3}}$ ).

In Fig. 3 we report two different outcomes of numerical simulations of the model, for two sets of parameters, in the left panel we found once again a consensus status, where all the population share the same opinion, as reported by the histogram. While in the right panel, the population polarizes ${ }^{\mathrm{b}}$ into clusters of different opinions, here 4 .

${ }^{\mathrm{b}}$ Let us observe that polarized case might be metastable; in fact if the mean separation 

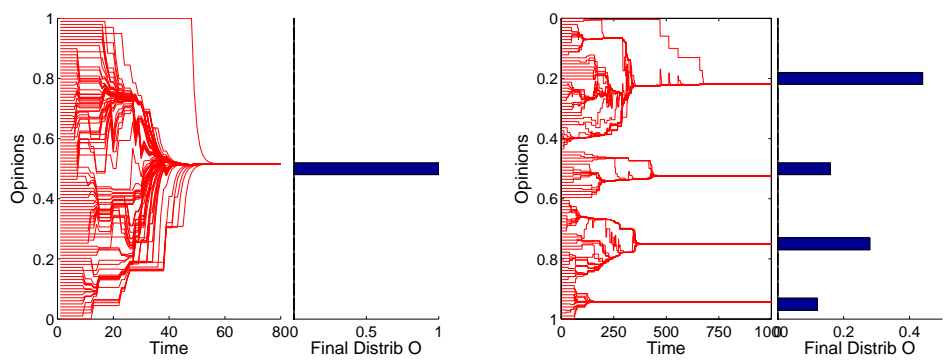

Fig. 3. Time evolution of the opinions and asymptotic opinion distribution. Parameters are: $\Delta g_{c}=0.1, \alpha_{c}=0.5$ (left panel), and $\Delta g_{c}=0.02, \alpha_{c}=0.5$ (right panel) both with $\Delta O_{c}=0.5$ and $N=100$ agents whose initial opinion are uniformly distributed in $[0,1]$, whereas initial affinities are uniformly distributed in $[0,1 / 2]$

Hence one can characterize the final asymptotic state with the number of opinion clusters as a function of the key parameters $\Delta g_{c}$ and $\alpha_{c}$. We observe that often the asymptotic state exhibits outliers, namely clusters formed by very few agents, and also that, because of the random encounters and initial distributions, the same set of parameters can produce asymptotic state that can exhibit a different number of opinion clusters. For this reason we define the average number of opinion clusters, $\left\langle N_{c l u}\right\rangle$, repeating the simulation a large number of times, here 500. A second possibility is to use the Deridda and Flyvbjerg number: ${ }^{12}$

$$
Y=\sum_{i=1}^{M} \frac{S_{i}^{2}}{N^{2}}
$$

where $M$ is the total number of clusters obtained in the asymptotic state and $S_{i}$ is the number of agents in the $i$-th cluster. The quadratic dependence on $S_{i} / N$ ensures that less weight has been given to small clusters with respect to larger ones.

In Fig. 4 we report the results of the analysis of $\left\langle N_{c l u}\right\rangle$ and $Y$ as a function of $\Delta g_{c}$, for a fixed value of $\alpha_{c}$.

between the adjacent opinion peaks is smaller than the opinion interaction threshold, $\Delta O_{c}$, and $\Delta g_{c}$ is not too small, there always exists a finite, though small, probability of selecting in the same acquaintance group individuals belonging to different opinion clusters, hence producing a gradual increase in the mutual affinities, which eventually lead to a merging of the, previously, separated clusters. This final state will be achieved on extremely long time scales, diverging with the group size: socially relevant dynamics are hence likely to correspond to the metastable regimes. A similar phenomenon has been observed in 6,10 . 

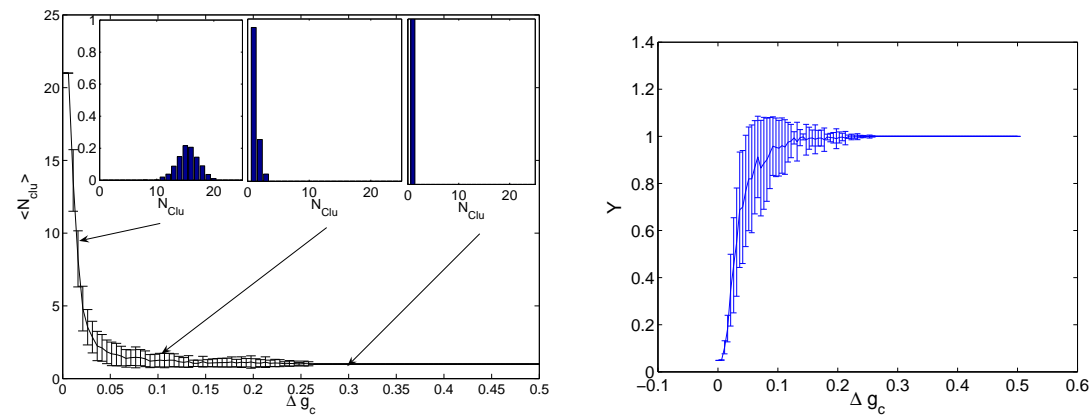

Fig. 4. Number of clusters (left panel) and Derrida and Flyvbjerg (right panel) as function of $\Delta g_{c}$ when $\alpha_{c}=0.5$. Average and Standard Deviation on 200 simulations. The distributions of the average number of cluster are presented for $\Delta g_{c}=0.3$ (right inset), $\Delta g_{c}=0.1$ (central inset) and $\Delta g_{c}=0.01$ (left inset)

A phase transition from a mono-cluster state, i.e. consensus, to polarization of the opinions in a population of 100 agents, emerges close to $\Delta g_{c}=0.25$, for smaller values of $\Delta g_{c}$ the distribution of the number of cluster can be well described by a normal distribution (see left inset Fig. 4), for larger value of $\Delta g_{c}$, only one cluster is present (see right inset Fig. 4), while for $\Delta g_{c}$ varying around 0.25 an exponential distribution can be found (see middle inset Fig. 4), reinforcing thus the statement of the existence of a phase transition.

Data from Fig. 4 suggest an exponential growth of $\left\langle N_{c l u}\right\rangle$ as a function of $\Delta g_{c}$ below the phase transition value, we thus compute a linear fit on $\log -\log$ scale (see Fig. 5) in the region involving small values of $\Delta g_{c}$, obtaining:

$$
\log <N_{\text {clu }}>=-1.495 \log \left(\Delta g_{c}\right)-4.107,
$$

when $\alpha_{c}=0.5$. A similar power law behavior is still valid also for the Derrida and Flyvbjerg number. The existence of a power low seems robust with respect to variations of the parameter $\alpha_{c}$ (see Fig. 5). The results presented in Fig. 5 allow us to extract also the behavior of the average number of clusters as a function of the second parameter $\alpha_{c}$ for a fixed $\Delta g_{c}$. In fact moving upward on vertical lines, i.e. decreasing $\alpha_{c}$, the $\left\langle N_{c l u}\right\rangle$ increases if $\Delta g_{c}$ is below the critical threshold, while above this value the number of clusters is always equal to one. Moreover from these data we could conclude that the phase transition point seems to be independent from the value of $\alpha_{c}$.

Another relevant quantity of interest, is the time needed to form an 


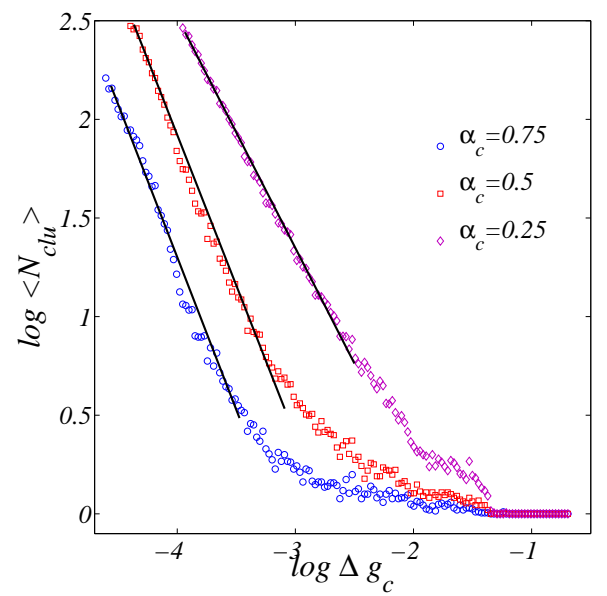

Fig. 5. Average number of clusters as function of $\Delta g_{c}$ (log-log scale). Best linear fits for different values of $\alpha_{c}$. Each simulation has been repeated 200 times

opinion cluster, the opinion convergence time, $T_{c}$, and its dependence of the size of the cluster. Numerical simulations not reported here, emphasize that in the polarization case $T_{c}$ depends in a highly non-trivial way on the total number of clusters and on their sizes, roughly speaking if in consensus state the time needed to form a cluster of say $N_{1}$ individual is some value $T_{1}$, then the time needed to form a cluster of the same size in a polarization case, has nothing to do with $T_{1}$ and it depends on all the formed clusters.

Because this paper offers a preliminary analysis, we decided to consider only the consensus case, hence choosing parameters ensuring the existence of only one cluster and we define the convergence time $T_{c}$ to be:

$$
T_{c}=\min \left\{t \geq 0: \max _{i}\left(O_{i}(t)\right)-\min _{i}\left(O_{i}(t)\right) \leq \frac{a}{N}\right\},
$$

where $a$ is a small parameter (hereby $a=0.1$ ).

We thus performed dedicated simulations with $N$ ranging from few unities to thousand unities. The results reported in Fig. 6 suggest a non-linear dependence of $T_{c}$ on $N$, well approximable by $T_{c} \sim N^{b}$. Using a regression analysis on the data, we can estimate the exponent which results, $b=0.091$ for $\Delta g_{c}=0.6$ and $b=0.087$ for $\Delta g_{c}=0.5$. Let us observe that as $\Delta g_{c}$ approaches the phase transition value, $T_{c}$ increases and the curve becomes more noisy (see for instance the bottom panel of Fig. 6 corresponding to $\Delta g_{c}=0.4$ ), that is because the occurrence of the consensus case becomes lesser and lesser probable. 

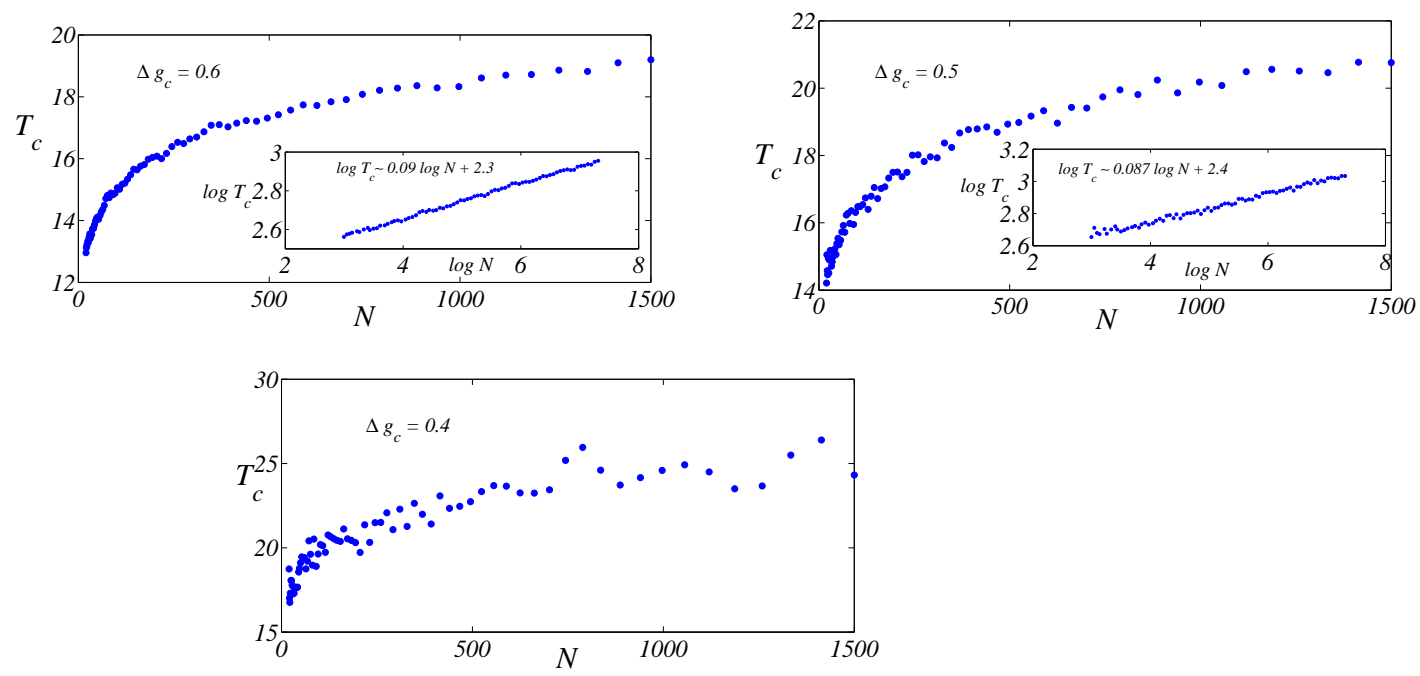

Fig. 6. Time of convergence of the opinions as function of number of agents. Panels correspond to parameters $\Delta g_{c}=0.6$ (top-left), $\Delta g_{c}=0.5$ (top-right) and $\Delta g_{c}=0.4$ (bottom). Insets $\log -\log$ plots of $T_{c}$ as a function of $N$.

\section{Conclusions}

In this paper we introduced a new model of opinion dynamics where agents meet in social groups, affinity driven, and possibly update their beliefs as a consequence of these local, i.e. group level, interactions. The model exhibits a rich phenomenology determined by the interlay between the dynamics of the opinions and the mutual affinities. We emphasized the role of two parameters, $\Delta g_{c}$ and $\alpha_{c}$, which can be qualitatively interpreted respectively as the openness of mind in the formation of the group and as the openness of mind in the intra-group dynamics. We thus studied the behavior of the model as a function of these two parameters.

The formulation of our model has been inspired by the observation of the way in which the formation mechanisms for social interactions do occur in the real world: a large majority of the processes of formation and evolution of the opinions are driven by group based discussions, such groups are determined by the mutual affinity and/or the shared opinion. The processes of group formation which tends to form clusters of acquaintances (or collaborators) are introduced in our model via the selection mechanism based on the mutual trust, i.e. Eq. (1) and (2).

The numerical analysis we performed, shows a dependence of the con- 
sensus/polarization state on the degree of mind openness in the creation of the groups of acquaintances, i.e. the parameter $\Delta g_{c}$, large values corresponding to consensus states while small ones to fragmented groups. That is the main reason why the model exhibits a phase transition with respect to this variable. Finally the intra-group dynamics, based on the mutual affinity, allows to update the opinions only for agents that perceive the group discussion close enough to its believes. This phenomenon is modeled by Eq. (5) and (6). Our analysis shows that the stronger is the degree of intra-group affinity required to make an interaction effective, i.e. large $\alpha_{c}$, the higher will be the degree of polarization of the population.

We can thus conclude that the model here presented, exhibits and well reproduces the two underlying dynamical mechanisms that can drive the opinion formation process in (relatively) small groups: exchange of information and mutual trust. Moreover these mechanisms evolve on different times scales as clearly showed previously.

This model represents thus a framework where to study groups interactions with applications to real social systems. It would be interesting to improve the model by introducing, at least, two factors: vectorial opinions $^{3,13,14}$ i.e. agents discuss and exchange information about more than one subject. Second, introduce a limitation in the number of agents with

which anyone can be affine with, as usually is the case in the real social networks.

\section{References}

1. S. Galam, arXiv:physics.sco-ph/0803.1800v1, (2008).

2. R. Hegselmann and U. Krause, JASSS, 5, no.3, (2002).

3. C. Castellano et al., preprint arXiv: 0710.3256, submitted Review of Modern Physics, (2008).

4. S. Galam and Y. Shapir, Math. J. Socio., 9, (1982), pp. 1.

5. G. Weisbuch et al, Complexity, 7, No. 3, 2002.

6. F. Bagnoli et al., Phys. Rev. E, 76, (2007), pp.066105.

7. S. Galam, Europhys. Lett., 70, (6), (2005), pp. 705.

8. S. Galam and F. Jacobs, Physica A, 381, (2007), pp. 366.

9. S. Galam, Physica A, 333, (2004), pp. 453.

10. T. Carletti et al., accepted proceedings WIVACE2008, (2008).

11. T. Carletti et al., Euro. Phys. J. B, in press, (2008).

12. B. Derrida and H. Flyvbjerg, J. Phys. A, 19, (1986), pp. 1003.

13. R. Axelrod, J. Conflict Resolution, 41, (1997), pp. 203.

14. S. Fortunato et Al., Int. Jour. Mod. Phys. C, 16, (2005),pp. 1535. 